\title{
INFLUENCIA DEL AGUA FREÁTICA SOBRE LA VEGETACIÓN DE LAS ÁREAS DE DESCARGA SOBRE ARENAS EN LA RESERVA BIOLÓGICA DE DOÑANA
}

\author{
José Carlos Muñoz Reinoso \\ Departamento de Biología Vegetal y Ecología. Universidad de Sevilla. Apdo. 1095. 41080 - Sevilla.
}

Palabras clave: hidroquímica, Doñana, acuífero, vegetación, CCA.

Keywords: groundwater hydrochemistry, Doñana, aquifer, vegetation, CCA

\begin{abstract}
.
INFLUENCE OF PHREATIC WATER TABLE ON THE VEGETATION OF AREAS OF DISCHARGE ON SAND IN THE DOÑANA BIOLOGICAL RESERVE.

The relationship between the vegetation of discharge areas on the sands of Donana Biological Reserve and both groundwater chemistry and depth of phreatic water table has been studied. Four types of waters have been detected each maintaining a defined plant community: Very mineralized waters (electrical conductivity $>1500 \mu \mathrm{S} / \mathrm{cm}$ ) related to Tamarix canariensis and Juncus acutus; mineralized waters $(500 \mu \mathrm{S} / \mathrm{cm})$ related to Scirpus holoschoenus and Juncus maritimus; little mineralized waters rich in chloride $(<500 \mu \mathrm{S} / \mathrm{cm})$ related to J.effusus, J. acutiflorus and Pteridium aquilinum; light mineralized waters $(<300 \mu \mathrm{S} / \mathrm{cm}) \mathrm{related}$ to vegetation which species composition depends on water table depth (deeper water table with Halimium halimifolium, Stauracanthus genistoides and Ulex australis, and shallow water table with Erica scoparia, Calluna vulgaris and Daphne gnidium).
\end{abstract}

\section{INTRODUCCIÓN}

El Parque Nacional de Doñana se asienta sobre el SE del sistema acuífero Almonte-Marismas, que tiene una extensión de $2400 \mathrm{~km}$, de los cuales el $75 \%$ se corresponden al acuífero libre, y el $25 \%$ restante se encuentra confinado bajo las arcillas de la marisma (I.G.M.E. 1983). El acuífero está constituido por depósitos marinos del Neógeno, cubiertos y parcialmente arrasados por sedimentos de origen fluvio-marino y eólico del Cuaternario. Como es esperado en un área costera sujeta a oscilaciones eustáticas importantes durante el Cuaternario, los materiales tienen una distribución heterogénea en detalle pero, en su conjunto, pueden ser considerado un gran acuífero (SUSO y LLAMAS 1993). La circulación del agua del acuífero se realiza principalmente en dirección Sur-Sureste (IGME 1983), lo que hace de la zona de arenas de Doñana un gran ecotono entre las zonas de recarga y las zonas de descarga del acuífero.

Los primeros estudios hidrogeológicos en el área de Doñana fueron realizados por la F.A.O. (1970) y el I.G.M.E. (1983) a finales de los años 60, proponiéndose el desarrollo del Plan de Transformación Agraria Alinonte-Marismas que consistía en la puesta en regadío de unas 24.000 ha. de marisma. Los primeros datos hidrogeoquímicos se publicaron en los años 80 (I.G.M.E. 1983, PLATA et al. 1983, BAONZA et al. 1984).

Más recientemente, estudios de carácter hidrológico han mostrado interés por el conocimiento del funcionamiento de los niveles freáticos, tanto en la zona saturada corno en la no saturada debido a la amenaza que supone la extracción de agua del acuífero para el mantenimiento de los humedales de Doñana (HOLLIS et al. 1989, SUSO LLAMAS 1990, CUSTODIO et al. 1992, OLÍAS 1995).

La vegetación de los humedales está determinada por procesos sucesionales y procesos ambientales (GONZALEZ BERNÁLDEZ \& MONTES 1989), de manera que la disponibilidad de agua en la zona no saturada y su calidad condicionan la distribución, abundancia y permanencia de las especies vegetales. Los estudios realizados por RAMÍREZ DÍAZ (1973), ALLIER et al. (1974) y RAMÍREZ DÍAZ et al. (1975) han definido distintos tipos de vegetación en las arenas estabilizadas según su composición, su estado sucesional y la profundidad del nivel freático. GONZÁLEZ BERNÁLDEZ et al. (1977) han estudiado la variación de la vegetación de la marisma de la Reserva Biológica en relación con los factores predominantes del medio, y MERINO et al. (1980) han tipificadoecológicamente 
la vegetación de la vera. Sin embargo, aunque existe conciencia de la importancia de las características del agua freática para la composición de la vegetación (GROOTJANS et al. 1988), se han realizado pocos trabajos sobre las relaciones entre la vegetación, la composición del agua freática y su dinámica (BERNALDEZ et al. 1990, REY BENAYAS et al. 1990, BERNALDEZ \& REY BENAYAS 1992).

El objetivo del presente trabajo es relacionar las comunidades de especies perennes (leñosas y helófitos) con las características fisicoquímicas del agua freática y determinar la heterogeneidad espacial de las mismas en zonas de descarga de la Reserva Biológica de Doñana.

\section{ÁREA DE ESTUDIO}

El Parque Nacional de Doñana se encuentra situado en el SO de España, en el extremo de la depresión del Guadalquivir y bañado por el océano Atlántico. Presenta tres grandes unidades ambientales y paisajísticas formadas por distintos materiales (arenas y arcillas): por un lado las dunas móviles y los cotos o arenas estabilizadas, y por otro lado la marisma (Fig. 1).

Las arenas de Doñana son de origen eólico, fundamentalmente cuarcíticas, estando la mayoría de los granos rodeados de una cutícula ferruginosa. El material subyacente presenta una composición muy semejante en cuanto a mineralogía y granulometría, indicando un mismo origen. Esta inalterabilidad del material original junto con el clima, son responsables de suelos escasamente desarrollados (entisoles e inceptisoles) de toda el área (SILJESTRÖM 1985).

El clima de Doñana es de tipo mediterráneo subhúmedo, con una precipitación media anual de $560 \mathrm{~mm}$ y una concentración del $80 \%$ entre Octubre y Marzo. Los meses de Noviembre y Diciembre son los más lluviosos, con medias de 91,1 y $92 \mathrm{~mm}$ respectivamente. Los inviernos son templados, con temperaturas medias de $9.3^{\circ} \mathrm{C}$ en los meses más fríos (Diciembre y Enero). En cambio, los veranos son muy secos, prácticamente sin precipitaciones en Julio y Agosto, y temperaturas medias de 23.4 y $23.6^{\circ} \mathrm{C}$ respectivamente (GARCIA NOVO 1979).

Doñana tiene una topografía ondulada reflejo de antiguos sistemas dunares hoy día parcialmente arrasados. La presencia de un nivel freático somero junto con la permeabilidad del sustrato hace las zonas más altas muy secas en verano, mientras que las depresiones pueden inundarse en invierno, dando lugar a innumerables lagunas. A la aparición y mantenimiento de estas lagunas contribuyen las descargas del

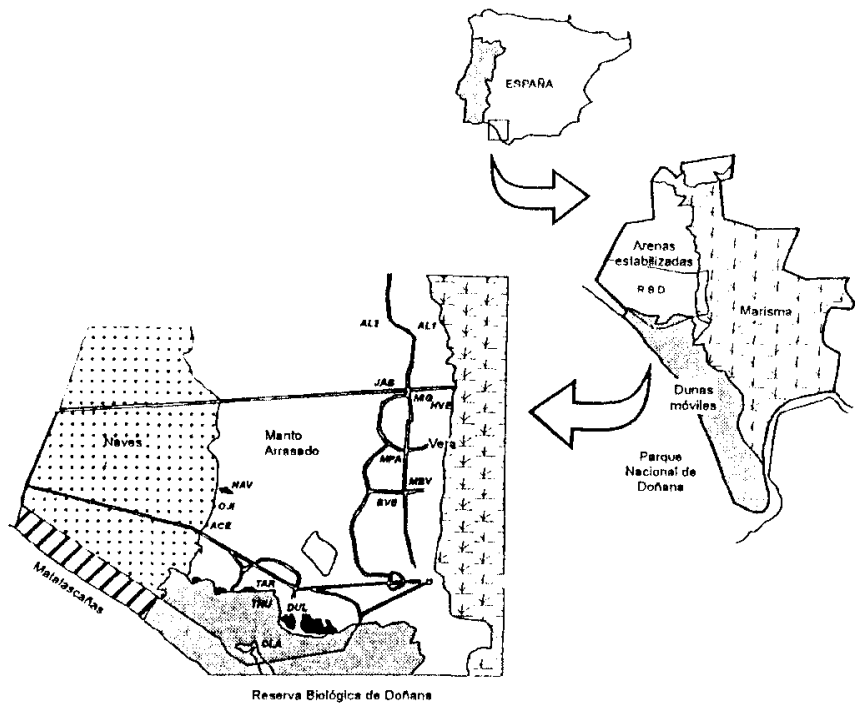

FIGURA 1. Localización dē los puntons de muestreo en el Parque Nacional de Doñana.

FIGURE 1. Location of sampling points in Doñana National Park.

Abreviaturas/Abbreviations: Navazo del Toro (NAV), Ojillo (OJI), Acebuche (ACE), Taraje (TAR), Taraje Nuevo (TNU), Corral Largo (CLA), Dulce (DUL), Brezal Vera (BVE), Monte Blanco Vera (MBV), Martín Pavón (MPA), Monte Intermedio Gangas (MIG), Helechal Vera (HVE), Jabata (JAB), Algaida 1 (ALI), Algaida 2 (AL2).

acuífero regional así como las descargas del acuífero dunar.

Las lagunas de Doñana han sido tipificadas por aspectos fisicoquímicos (GARCIA NOVO et al. 1991) y morfométricos (BRAVO \& MONTES 1993). Las lagunas de mayor entidad han sido caracterizadas limnológicamente (LOPEZ et al. 1991, TOJA et al. 1991, SERRANO \& TOJA 1995), mostrando marcadas diferencias anuales e interanuales. En general, se trata de cuerpos de agua relativamente pequeños, de aguas alcalinas ricas en sodio y en constante interacción con las aguas subterráneas (SACKS et al 1992, LÓPEZ et al. 1994).

El agua subterránea en la zona del acuífero libre es, en general, poco mineralizada y de tipo clorurado sódico (GUIMERA et al. 1991, MANZANO et al. 1991, LÓPEZ et al. 1994, OLÍAS 1995). La zona de mayor mineralización se localiza en el contacto del acuífero libre y el confinado. La conductividad, que muestra una alta correlación con cloruros y sodio, es baja (unos $500 \mu \mathrm{S} / \mathrm{cm}$ de media) excepto en las zonas de descarga local y en las zonas influidas por lagunas importantes (VELA 1984, LOPEZ et al. 1994); sus valores más elevados se registran en los períodos de recarga (inviernoprimavera) y los menores en verano (LÓPEZ et al. 1994, OLÍAS et al. 1994, OLÍAS 1995).

La vegetación actual, cuya composición florística depende de la disponibilidad hídrica, está dominada por el matorral mediterráneo, resultado de la degradación del bosque original (RAMIREZ DIAZ et al. 1975). Las zonas más altas están 
dominadas por un matorral xerófilo, compuesto principalmente por cistáceas y labiadas. En las zonas bajas domina el brezal. Las lagunas pueden aparecer orladas por densos helechales de Pteridium aquilinum (L.)Kuhn, mientras que el borde más interno aparece cubierto por juncos de distintas especies (J. acutus L., J. maritimus Lam., J. effusus L., Scirpus holoschoenus L.). Las comunidades de macrófitos acuáticos son ricas en especies, reflejando la variedad de ambientes acuáticos que existen en el Parque en cuanto al sustrato de las cubetas, su funcionamiento hidrológico, amplitud y fluctuaciones de los gradientes de salinidad y estado trófico de las aguas (GARCIA MURILLO et al. 1993), apareciendo especies de los géneros Chara, Nitella, Ricciocarpus, Ranunculus, Elatine, Miriophyllum, etc. Cuando desaparece la lámina de agua, la laguna es colonizada por pastizales de terófitos anuales.

\section{MATERIAL Y MÉTODOS}

Con la ayuda de la fotografía aérea y recorridos previos del terreno se eligió un conjunto de puntos de muestreo localizados en áreas de descarga de la zona de arenas. Estas áreas de descarga se encuentran localizadas en zonas de transición entre las distintas unidades ambientales y geomorfológicas de la Reserva Biológica de Doñana. Estas tres grandes zonas son la transición entre las Naves (área topográficamente más elevada de las arenas estabilizadas) y el Manto Arrasado, la transición entre las dunas móviles y las arenas estabilizadas y en la proximidad de la transición entre las arenas estabilizadas y la marisma. De esta forma, se eligieron 15 puntos de muestreo que mostraban distintos tipos de vegetación presentes en el área de estudio (Fig. 1). Se realizó un total de tres muestreos durante la primavera-verano de 1994.

Las muestras de agua fueron extraídas mediante una bomba peristáltica manual de piezómetros de $40 \mathrm{~mm}$ de diámetro y 1.5-2.5 m de profundidad instalados en los puntos de muestreo dentro de un programa de estudio más amplio. Previamente a la toma de la muestra se registró el nivel piezométrico (PNF). $\mathrm{El} \mathrm{pH}$, la conductividad (Con) y la temperatura se midieron in situ mediante $\mathrm{pH}$-metro y conductivímetro portátiles de la marca CRISON, calibrados previamente a la lectura. Las muestras de agua se guardaron herméticamente protegidas de la luz en frío hasta su traslado al laboratorio, donde fueron conservadas a $4^{\circ} \mathrm{C}$. El agua fue sometida a análisis químico, determinándose los siguientes parámetros: calcio $\left(\mathrm{Ca}^{21}\right)$, magnesio $\left(\mathrm{Mg}^{2+}\right)$, sodio $\left(\mathrm{Na}^{+}\right)$y potasio $\left(\mathrm{K}^{\prime}\right)$ mediante absorción atómica, y sulfatos $\left(\mathrm{SO}_{4}{ }^{2}\right)$ y cloruros $\left(\mathrm{Cl}^{-}\right)$mediante el método APHA (1985). En el tercer muestreo también se determinó la rcscrva alcalina total (CA)(APHA 1985).
La vegetación fue muestreada en parcelas de 10 x 10 m' adyacentes al piezómetro instalado en cada punto de muestreo. En cada parcela se registró la presencia de especies perennes (leñosas, helófitos y helechos). A cada especie se le asignó un valor de 1 a 5 según su cobertura de acuerdo con la siguiente escala: 1) menos del $5 \%$ de cobertura; 2) de 5-25\%; 3) de 25 $50 \%$; 4) de 50-75\%; y 5) más del 75\%, eliminándose las especies que aparecían en una sola parcela. De esta forma se obtuvo una matriz de 15 parcelas x 18 especies.

Las muestras del agua freática y las de vegetación fueron sometidas a análisis de clasificación aglomerativo utilizando en el primer caso la distancia euclídea y en el segundo el porcentaje de similaridad como medidas de similaridad.

Los datos conjuntos de profundidad y fisico-química del agua freática y vegetación fueron sometidos a un Análisis Canónico de Correspondencias (CCA) mediante el programa CANOCO (ter BRAAK 1991). Previamente, las variables del agua freática fueron transformadas como $\mathrm{x}^{\prime}=\log (\mathrm{x}+1)$ para aproximarlas a distribuciones normales. El CCA permite comprobar estadísticamente si las especies están relacionadas con variables ambientales y ver la importancia relativa de estas variables para explicar la distribución de las especies, puesto que sus ejes de ordenación están restringidos a ser combinación lineal de las variables ambientales (ter BRAAK 1987). Dos variables ambientales (conductividad y sulfatos) fueron excluidas del análisis para evitar efectos de multicolinearidad con el resto de las variables. Además, dos muestras con altos contenidos iónicos (con 15 veces más cloruros y 10 veces más sodio que la media de las restantes muestras) se hicieron pasivas, es decir, se incluyeron en la ordenación pero sin influencia en la extracción de los ejes de ordenación.

\section{RESULTADOS}

La Tabla 1 muestra los datos fisicoquímicos y de profundidad del agua freática en los distintos puntos de muestreo. Destacan Navazo del Toro (NAV) y Taraje Nuevo (TNU) con altos valores de conductividad y de concentraciones iónicas, frente a aguas ligeramente mineralizadas como las del Corral Largo, así como los altos contenidos de carbonatos en las lagunas de mayor extensión y zonas adyacentes (Dulce, Taraje, Navazo y Taraje Nuevo). Los valores de pH están comprendidos entre 6.5 en Algaida 2 (AL2) y 7.5 en TNU y el nivel freático se encontró a una profundidad de entre $88 \mathrm{~cm}$ en Taraje y 213 en el monte blanco de la vera (MBV) en Julio.

La Fig. 2 muestra la clasificación de las muestras según las características fisicoquímicas y de profundidad de su agua 
TABLA 1. Características fisicoquímicas medias y profundidad del agua freática en Julio de los puntos de muestreo. Conductividad a 25"C. CA: reserva alcalina total. PNF: profundidad del nivel freático.

TABLE 1. Mean water table physical and chemical characteristics and depth (July) of sampling points. Conductivity at $25^{\circ} \mathrm{C} \mathrm{CA:} \mathrm{total} \mathrm{alkalinity.} \mathrm{PNF:} \mathrm{water} \mathrm{level} \mathrm{depth.}$

\begin{tabular}{|c|c|c|c|c|c|c|c|c|c|c|}
\hline & $\mathrm{pH}$ & $\begin{array}{c}\text { Con } \\
(\mu \mathrm{S} / \mathrm{cm})\end{array}$ & $\underset{(\mathrm{meq} / 1)}{\mathrm{Ya}}$ & $\underset{(\mathrm{meq} / \mathrm{l})}{\mathrm{K}}$ & $\begin{array}{c}\mathrm{Mg} \\
(\mathrm{meq} / \mathrm{l})\end{array}$ & $\underset{(\mathrm{meq} / 1)}{\mathrm{Ca}}$ & $\begin{array}{c}\mathrm{SO}, \\
(\mathrm{meq} / \mathrm{l})\end{array}$ & $\underset{(\mathrm{meq} / \mathrm{l})}{\mathrm{Cl}}$ & $\underset{(\mathrm{meq} / \mathrm{l})}{\mathrm{CA}}$ & $\underset{\text { (m) }}{\operatorname{PNF}(\text { Julio) }}$ \\
\hline $\mathrm{TNU}$ & 75 & 2237 & 1336 & 043 & 352 & 360 & 192 & 7493 & 640 & 181 \\
\hline NAV & 71 & 1622 & 1208 & 084 & I 57 & 113 & 323 & 6229 & 346 & 125 \\
\hline OJI & 68 & 204 & 102 & 067 & 014 & 018 & 068 & 254 & 151 & 135 \\
\hline ACE & 68 & 702 & 079 & 040 & 019 & 020 & 144 & 200 & 087 & 166 \\
\hline TAR & 69 & 318 & 121 & 035 & 009 & 018 & 078 & 383 & 332 & 088 \\
\hline DUL & 71 & 565 & 288 & 040 & 052 & $0 \mathrm{~S} 5$ & 346 & 287 & 390 & 125 \\
\hline CLA & 67 & 145 & 058 & 044 & 004 & 041 & 094 & 287 & 090 & 179 \\
\hline AL 1 & 73 & 478 & 106 & 058 & 020 & 032 & 168 & 326 & 1.15 & 097 \\
\hline HVE & 71 & 405 & 160 & 044 & 038 & 048 & 228 & 387 & 089 & 180 \\
\hline AL2 & 65 & 260 & 106 & 037 & 016 & 022 & 101 & 433 & O62 & 113 \\
\hline JAB & 67 & 228 & 106 & 043 & 019 & 018 & 071 & 512 & 067 & 132 \\
\hline MPA & 66 & 168 & 045 & 047 & 008 & 013 & 051 & 456 & 083 & 107 \\
\hline BVE & 68 & 158 & 058 & 031 & 007 & 009 & 048 & 328 & 039 & 166 \\
\hline MIG & 68 & 412 & 212 & 040 & 030 & 035 & 109 & 534 & 042 & 134 \\
\hline MBV & 70 & 246 & 085 & 035 & 018 & 028 & 101 & 292 & 040 & 213 \\
\hline
\end{tabular}

freática. Pueden distinguirse 4 grupos, dos más nítidos y otros dos con características muy similares:

a) Taraje Nuevo (TNU) y Navazo del Toro (NAV) con aguas muy mineralizadas, con altos contenidos en todos los iones, especialmente en cloruros y sodio;

b) Taraje (TAR), Dulce (DUL) y Algaida 1 (ALI), con aguas mineralizadas caracterizadas por valores altos de carbonatos;

c) Este grupo incluye 5 puntos de muestreo, Helechal Vera (HVE), Monte Blanco Vera (MBV), Acebuche (ACE), Brezal Vera (BVE) y Corral Largo (CLA), presenta aguas entre escasa y ligeramente mineralizadas con los niveles freáticos más profundos;

d) Este grupo, con los 5 puntos de muestreo restantes, Ojillo (OJI), Jabata (JAB), Algaida 2 (AL2), Martín Pavón (MPA) y Monte Intermedio de las Gangas (MIG), se diferencia del anterior fundamentalmente por presentar niveles freáticos más someros y mayores contenidos en cloruros.

La Figura 3 muestra el resultado del análisis de clasificación de los puntos muestreados según la composición de su vegetación. Pueden distinguirse 4 grupos:

a) Compuesto por Ojillo, Algaida2, Acebuche y Brezal Vera, y caracterizado por una vegetación de monte negro, con Erica scoparia L., Calluna vulgaris (L.)Hull, Daphne gnidium L. Rubus ulmifolius Schott y Cistus salvifolius L.;

b) Con Algaidal, Jabata, Martín Pavón y Helechal de la Vera, con una vegetación compuesta por Juncus acutiflorus Ehrh., J. effusus, Cyperus longus L.. Salix atrocinerea Brot.y Pteridium aquilinum; c) Monte Intermedio de las Gangas, Monte Blanco Vera y Corral Largo, con una vegetación con Ulex australis Clemente, Halirnium halimifolium (L.) Willk., Stauracanthus genistoides (Brot.)Sampaio y Pinus pinea L.;

d) Taraje Nuevo, Navazo del Toro, Taraje y Dulce, con una vegetación caracterizada por la presencia de Juncus acutus y $J$. maritimus con Scirpus holoschoenus y Tamarix canariensis (Willd.).

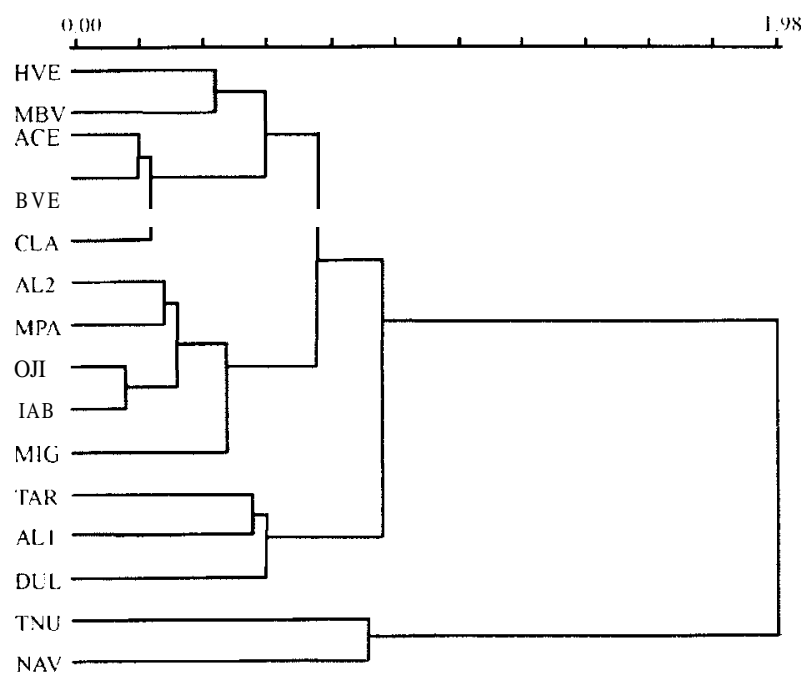

FIGURA 2. Clasificación de los puntos de muestreo según las características del agua freática. Abreviaturas como en Fig. 1

FIGURE 2. Result of Cluster Analysis of sampling points based on phreatic water characteristics. Abbreviations as Fig 1 
La Tabla 2 resume el resultado final del Análisis Canónico de Correspondencias (CCA) de 15 muestras, con 17 especies y 8 variables ambientales. La Figura 4 es la representación gráfica del resultado del CCA. El primer eje, que absorbe un $31.8 \%$ de

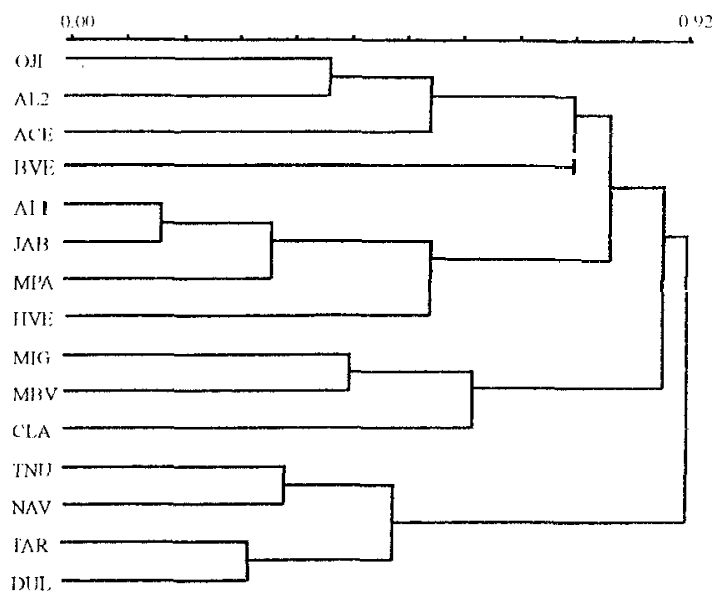

FIGURA 3. Clasificación de los puntos de muestreo según su vegetación. Abreviaturas como Fig. 1 .

FIGURE 3. Result of Cluster Analysis of sampling points based on vegetation composition. Abbreviations as Fig. 1.

la varianza, está correlacionado positivamente con el contenido en carbonatos y en sodio, y negativamente con el contenido en cloruros; representa fundamentalmente un gradiente de mineralización (alcalinidad y halofitismo). En el extremo positivo del primer eje se ordenan las parcelas localizadas en las lagunas de mayor superficie, situadas fundamentalmente delante del frente de dunas móviles. Las especies que se sitúan en este extremo son Juncus acutus, J. maritimus y Scirpus holoschoenus. En un análisis de correspondencias previo, que no se incluye por la similaridad en los resultados, aparece asociado a este grupo Tamarix canariensis, que no aparece en el CCA por haberse hecho pasivas las dos muestras que lo incluían (Navazo del Toro y Taraje Nuevo).

TABLA 2. Resultados del Análisis Canónico de Correspondencias. TABLE 2. Summary results of the Canonical Correspondece Analysis

\begin{tabular}{|c|c|c|c|c|}
\hline & EJE I & EJE II & EJE III & EJE IV \\
\hline$\overline{\text { Autuvalores }}$ & 0793 & 0670 & 0.353 & 0313 \\
\hline Correlaciones especies-var ambientales & 0997 & 0947 & 0963 & 0880 \\
\hline Varianza explicada (especies) & 238 & 20.1 & 106 & 94 \\
\hline Varianza explicada (especies-v ainbientales) & 318 & 269 & 142 & 126 \\
\hline \multicolumn{5}{|l|}{ Corretaciones variables ambientales-ejes } \\
\hline $\mathrm{Na}$ & 049 & 006 & -040 & 050 \\
\hline $\mathrm{K}$ & -021 & -036 & 045 & 008 \\
\hline $\mathrm{Mg}$ & 039 & -004 & -030 & 054 \\
\hline $\mathrm{Ca}$ & 033 & 028 & 007 & 07 \\
\hline $\mathrm{Cl}$ & -0.46 & -027 & -015 & 012 \\
\hline $\mathrm{CA}$ & 0.87 & -0.24 & 008 & 0.31 \\
\hline PNF & -018 & 0.71 & -007 & -0 02 \\
\hline
\end{tabular}

En el extremo negativo del primer eje se encuentra un grupo heterogéneo de parcelas compuesto por pequeñas lagunas y matorrales próximos a la vera, compuestos fundamentalmentepor Pteridium aquilinum y las especies más glicófitas (J. effusus, J. acutiflorus (Ehrh.), Cyperus longus (L.), Salix atrocirzerea (Brot.).

El segundo eje, que absorbe un $26.9 \%$ de la varianza, está correlacionado positivamente con la profundidad del nivel freático y puede interpretarse como un gradiente de higrofitismo. Separa especies no freatófitas o de monte blanco en sentido amplio (Halimium halimifolium, Stauracanthus genistoides, Ulex australis, Pinus pinea), de especies freatófitas, asociadas a depresiones y lagunas (Salix sp, Tamarix sp, Pteridium aquilinum, Juncus sp). En el centro del diagrama aparecen las especies higrófitas terrestres o de monte negro (Erica scoparia. Calluna vulgaris. Daphne gnidium, Rubus ulmifolius, Cistus salvifolius).

\section{DISCUSIÓN}

Los resultados obtenidos indican que existe una estrecha relación entre la profundidad, las características fisicoquímicas del agua freática y la vegetación en el área de Doñana. Trabajos similares han demostrado la relación entre la vegetación existente y la hidroquímica del agua freática en otras zonas (REY BENAYAS et al. 1990, BOEYE \& VERHEYEN 1994).

En el presente estudio se han detectado distintos tipos de aguas subterráneas que reflejan la heterogeneidad espacial existente en el sustrato de Doñana y que modifican las características generales del agua de la zona no saturada en la zona del acuífero libre. De esta forma, se han detectado:

a) aguas muy mineralizadas por influencia de horizontes edáficos subsuperficiales muy salinos, como es el caso de Navazo del Toro y Taraje Nuevo (conductividad $>1500 \mu \mathrm{S} / \mathrm{cm}$ );

b) aguas mineralizadas $(500 \mu \mathrm{S} / \mathrm{cm})$ influidas por las aguas de lagunas próximas debido a la entidad de estos cuerpos de agua y a los procesos biológicos que allí tienen lugar, como es el caso de Taraje y Dulce;

c) aguas poco mineralizadas $(<500 \mu \mathrm{S} / \mathrm{cm})$ con importante presencia de cloruros debido a la proximidad a la marisma (MANZANO et al. 1991), como las pequeñas lagunas de La Jabata, Martín Pavón y La Algaida; y

d) aguas ligeramente mineratizadas $(<300 \mu \mathrm{S} / \mathrm{cm})$, presentes en zonas que funcionan fundamentalmente como zonas de recarga y temporalmentecomo zonas de descarga, como los corrales dunares.

Asociadas a la disponibilidad del agua y a sus características fisicoquímicas, los análisis han permitido la detección de 4 comunidades vegetales distintas: por un lado, dos comunidades 


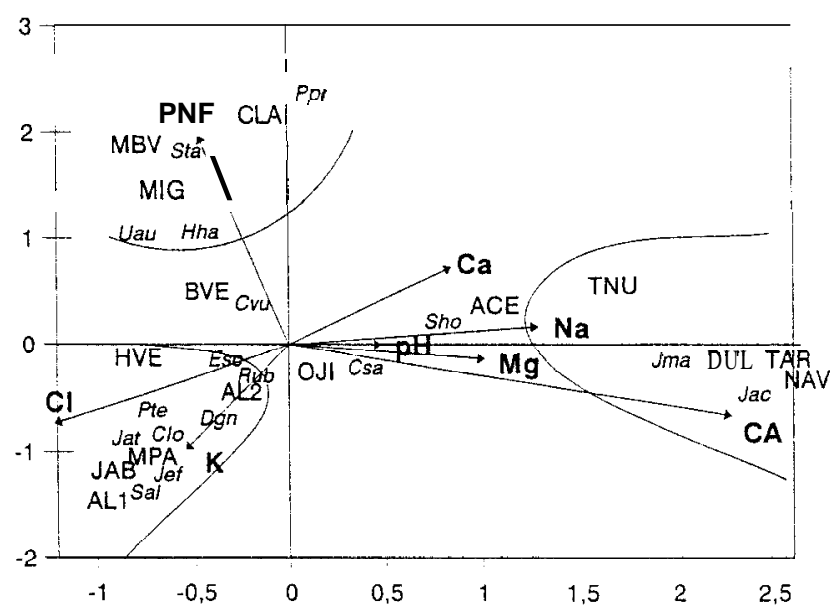

FIGURA 4 Análisis Canónico de Correspondencias. Ordenación conjunta de las especies, los puntos de muestreo y las variables ambientales.

FIGURE 4. Canonical Correspondence Analysis. Ordination of species, sampling points and environmental variables.

Especies/Species: Cvu: Calluna vulgaris; Csa: Cistus salvifolius; Clo: Cyperus Longus; Dgn: Daphne gnidium; Esc: Erica scoparia; Hha: Halimium halimifolium,; Jat: Juncus acutiflorus,; Jac: J. acutus; Jef: J. effusus; Jma: J. maritimus; Ppi: Pinrrs pinea; Pte: Pteridium aquilinum; Rub: Rubus ulmifolius; Sal: Salix atrocinerea, Sci: Scirpus holoschoenus; Sta: Stauracanthus genistoides; Uau: Ulex australis.

de especies no freatófitas con distinto grado de higrofitismo y, por otro lado, dos comunidades freatófitas con distinto grado de halofitismo. Estos grupos detectados se corresponden a su vez a 7 asociaciones fitosociológicas.

El primer grupo está formado por especies terrestres xerófitas, representadas por Halimium halimifolium, Stauracanthus genistoides y Ulex australis, que se localizan en lugares generalmente no encharcables, a los que subyace un freático con aguas poco mineralizadas y de baja conductividad $(<300$ $\mu \mathrm{S} / \mathrm{cm}$ ). Esta comunidad se correspondería con el monte blanco de RAMIREZ DIAZ (1973) o el Halimio halimifoliiStauracanthetum genistoidis de RIVAS MARTINEZ et al. (1980).

El segundo grupo está compuesto por especies terrestres higrófitas como Calluna vulgaris, Erica scoparia, Rubus ulmifolius, Cistus salvifolius y Daphne gnidium. El agua freática tiene características fisicoquímicas similares a las del grupo anterior, pero es más somero $(<1.70 \mathrm{~m}$ en Julio de 1994). Se corresponde con el monte negro de RAMIREZ DIAZ (1973) o el Erico scopariae-Ulicetum australis de RIVAS MARTINEZ et al. (1980).

El tercer grupo está compuesto por especies como Pteridium aquilinum, Cyperus longus, Juncus effusus, J. acutiflorus y Salix atrocinerea, acompañadas en los lugares más conservados e higrófilos por Erica ciliaris y Ulex minor. Se trata de juncales higrófilos de pequeñas lagunas de agua dulce, con leñosas quc soportan el encharcamiento tcmporal, con aguas de conductividades menores a $500 \mu \mathrm{Slcm}$ y contenidos relativamente altos en cloruros. Se corresponde con el Juncetum rugoso-effusi y Erico ciliaris-Ulicetum lusitanici de RIVAS MARTINEZ et al. (1980) y los juncales con monte negro higrofítico de RAMIREZ DIAZ (1973).

El cuarto grupo está compuesto por J. acutus, J. maritimus y Scirpus holoschoenus, especies presentes en lagunas de mayor superficie y relacionadas con aguas subterráneas mineralizadas (GONZALEZ BERNALDEZ \& MONTES 1989), aunque según RIVAS MARTINEZ et al. (1980) J. acutus, J. maritimus y Scirpus holoschoenus se asocian a comunidades no halófilas en el área de Doñana. Se corresponde con las comunidades Galio palustri-Juncetum maritimi y HoloschoenoJuncetum acuti. En general, se trata de aguas con conductividades superiores a $500 \mu \mathrm{Slcm}$ y contenidos altos en carbonatos. Según LOPEZ et al (1994), esto último puede ser debido a la actividad fotosintética de las lagunas, cuyas aguas se mezclan con las del acuífero, como han demostrado VELA et al. (1987).

Mención aparte merecen Navazo del Toro y Taraje Nuevo, únicos puntos muestreados donde aparecen tarajes (Tamarix canariensis), con aguas freáticas con conductividades superiores a $1500 \mu \mathrm{S} / \mathrm{cm}$ y altos contenidos en cloruros y sodio, lo cual puede ser debido a que en ambos emplazamientos subyacen lagunas enterradas, como ha indicado SILJESTRÖM (1985) para Navazo del Toro estudiando su suelo y GARCÍA SÁNCHEZ (com. pers.) para Taraje Nuevo al encontrar restos de diatomeas en el sedimento. La asociación fitosociológica correspondiente sería la Polygono-Tamaricetum africanae.

Los resultados obtenidos sobre las características del agua freática de la zona no saturada están en consonancia con los obtenidos por otros autores en la misma área, en la que se dan sistemas de flujos regionales, semilocales y locales (VELA 1984, LÓPEZ et al. 1994) y existe una gran heterogenidad espacial en el sustrato debido a la génesis e historia reciente del territorio.

En el área de Doñana, las comunidades vegetales pueden asociarse a las características hidroquímicas del agua de la zona no saturada. La detección de alteraciones en la vegetación puede indicar alteraciones importantes en la disponibilidad y en las características fisicoquímicas del agua freática, adquiriendo las especies un valor como indicador biológico (BERNALDEZ et al. 1990).

\section{AGRADECIMIENTOS}

Dolores Jordano realizó los análisis de agua. Agustín Cortés solucionó las dudas taxonómicas. El Dr. García Novo realizó una lectura crítica del manuscrito y la Dra. Toja realizó 
interesantes sugerencias. La colaboración de J.B. Gallego fue fundamental para la realización de la Figura 1.

\section{BIBLIOGRAFÍA}

ALLIER, C.; F. GONZÁLEZ BERNÁLDEZ y RAMÍREZ 1974. Mapa ecológico de la Reserva Biológica de Doñana.C.S.I.C. Estación Biológica de Doñana.

APHA 1985. Standard methods for examination of water and wastewater. 16th Ed. Am. Publ. Health Assoc. Washington D.C.

BAONZA, E.; A. PLATA y A. SILGADO 1984. Hidrología isotópica de las aguas subterráneas del Parque Nacional de Doñana y su zona de-influencia. Cuadernos de Investigación. C7.CEDEX. Madrid. 139 pp.

BERNÁLDEZ, F.G.; J.M. REY BENAYAS, B. PECO \& C. LEVASSOR 1990. Groundwater indicators plants in the tertiary aquifers of central Spain. Hydrogeology, Selected Papers. Vol. 1: 301-312.

BERNÁLDEZ, F.G. \& J.M. REY BENAYAS 1992. Geochemical relationships between groundwater and wetland soils and their effects on vegetation in central Spain. Geoderma 55: 273-288.

BOEYE, D. \& R.F. VERHEYEN 1994. The relation between vegetation and soil chemistry gradients in a ground water discharge fen. J. Veg. Sci. 5: 553-560.

BRAVO, M.A. y C. MONTES 1993. Inventario de las formaciones palustres del manto eólico del Parque Nacional de Doñana (SW Spain). Actas VI Congreso Español de Limnología, Granada: 31-43.

CUSTODIO, E.; J. DOLZ, J. GUIMERÁ, M. MANZANO, R. PONCELA, J. SAM PER, M. SÁNCHEZ y E. VELASCO 1992. Aportaciones al conocimiento hidrogeológico de los acuíferos del Parque Nacional de Doñana y su entorno. Hidrogeologia y Recursos Hidráulicos. XVI: 425-439.

F.A.O. 1970. Estudio hidrogeológico de la cuenca del Guadalquivir: informe técnico. 1 AGL. SF/SPA 9, Roma: 115 pp (informe inédito).

GARCÍA MURILLO, P.; M. BERNÚES y C. MONTES 1993. Los macrófitos acuáticos del Parque Nacional de Doñana (SW España). Aspectos florísticos. Actas VI Congreso Español de Limnología, Granada: 261-267.

GARCÍA NOVO, F. 1979. The ecology of vegetation of the dunes in Doñana National Park (South West Spain). In: Jefferies, R.L. and A.J. Davy (ed) Ecological Processes in Coastal Environments. Blackwell, Oxford, pp 571-592.

GARCÍA NOVO, F.; D. GALINDO, J.A. GARCÍA SÁNCHEZ, C. GUISANDE, J. JÁUREGUI, T. LÓPEZ, N. MAZUELOS, J.C. MUÑOZ, L. SERRANO y J.
TOJA 1991. Tipificación de los ecosistemas acuáticos sobre sustrato arenoso del Parque Nacional de Doñana. Actas III Simposio del Agua en Andalucia, Córdoba: 165-176.

GONZÁLEZ BERNÁLDEZ, F.; L. RAMÍREZ, A. TORRES y F. DÍAZ PINEDA 1977. Estructura de la vegetación de marisma de la Reserva Biológica de Doñana (Huelva). II. Estudio de un gradiente de salinidad. Anales Edaf. Agrob. 36 (9-10): 1005-1017.

GONZÁLEZ BERNÁLDEZ, F. y C. MONTES (coord.) 1989. Los humedales del acuifero de Madrid. Inventario y Tipologia basada en su origen y funcionamiento. Canal de Isabel II. Madrid. 92 pp.

GROOTJANS, A.P.; R. van DIGGELEN, M.J. WASSEN \& W.A. WERSINGA 1988. The effects of drainage on groundwater quality and plant species distribution in stream valley meadows. Vegetatio 75: 37-48.

GUIMERÁ, J.; E. CUSTODIO y L. CANDELA 1991. Caracterización de la recarga de los acuíferos mediante trazador químico artificial en el Parque Nacional de Doñana (Huelva, España). Rev. Geofisica 47: 135-147.

GUIMERÁ, J.; E. CUSTODIO y L. CANDELA 1991. Caracterización de la recarga de los acuíferos mediante trazador químico artificial en el Parque Nacional de Doñana (Huelva, España). Rev. Geofísica 47: 135-147.

HOLLIS, T.; J. MERCER and P. HEURTEAUX 1989. The implications of groundwater entractiorz for the longterm future of the Doñana National Park. Report of the WWF/UICN/ADENA. Mission to Doñana National Park, November 1988,60 pp.

I.G.M.E. 1983. Hidrogeologia del Parque Nacional de Doñana y su Entorno. Serv. Publ. Min. Industria y Energía. Madrid. $120 \mathrm{pp}+1$ mapa.

LÓPEZ, T.; N. MAZUELOS y J.C. MUÑOZ REINOS0 1994. Spatial and temporal variation in the ionic composition of shallow water table in Doñana National Park (SW Spain). Verh. Internat. Verein. Limnol. 25: 1438-1444.

LÓPEZ, T.; J. TOJA \& N. GABALLONE 1991. Limnological comparison of two peridunar ponds in the Doñana National Park (Spain). Arch. Hydrobiol. 120: 357-378.

MANZANO, M.; E. CUSTODIO y R. PONCELA 1991. Contribución de la hidrogeoquímica al conocimiento de la hidrodinámica de los acuíferos en el área de Doñana. Actas 111 Simposio del Agua en Andalucía, Córdoba: 457-486.

MERINO, J.; L. RAMÍREZ, F. SANCHO y A. TORRES 1980. Estudio ecológico de la vegetación de ecotono (vera) de la Reserva Biológica de Doñana (Huelva). II Tipificación ecológica de las comunidades vegetales. Anales 
Edaf. Agrob. 39(11-12): 1867-1878.

OLÍAS, M. 1995. Evaluación de la recarga y comportamiento de la zona no saturada del acuifero Almonte-Marismas (Huelva).Tesis Doctoral. Univ. Granada.

OLÍAS, M.; J. CRUZ SAN JULIÁN, J. BENAVENTE y C. ALMEIDA 1994. Evolución hidroquímica temporal en algunos puntos de observación en el acuífero AlmonteMarismas (Huelva). Bol. Geol. Min. Vol 105-4: 362-371.

PLATA, A.E., BAONZA y A. SILGADO 1983. Hidrología isotópica de las aguas subterráneas del Parque Nacional de Doñana y su zona de influencia. Isotopes in Hydrology. IAEA.SM-270/57. Viena: 321-340.

RAMÍREZ DÍAZ, L. 1973. Estudio ecológico cuantitativo del matorral de la Reserva Biológica de Doñana. Tesis Doctoral. Univ. de Sevilla. 394 pp.

RAMÍREZ DÍAZ, L.; F. GARCÍA NOVO, J. MERINO ORTEGA y F. GONZÁLEZ BERNÁLDEZ 1975 Sistemas de dunas y arenas estabilizadas de la Reserva Biológica de Doñana. En: Doñana. Prospección e inventario de ecosistemas: 159-193. Monografía 18. ICONA-MAPA. Madrid.

REY BENAYAS, J.M.; F.G. BERNÁLDEZ, C. LEVASSOR \& B. PECO 1990. Vegetation of groundwater discharge sites in the Douro basin, central Spain. J. Veg. Sci. 1: 461-466.

RIVAS MARTÍNEZ, S.; M. COSTA, S. CASTROVIEJO y E. VALDÉS 1980. Vegetación de Doñana (Huelva, España). Lazaroa 2: 1-189 pp.

SACKS, L.A.; J.S. HERMAN, L.F. KONIKOW and A.L. VELA 1992. Seasonal dynamics of groundwater-lake interactions at Doñana National Park, Spain. J. Hydrology
136: 123-154.

SERRANO, L. \& J. TOJA 1995. Limnological description of four temporary ponds in the Doñana National Park (SW Spain). Arch. Hydrobiol. 133: 497-516.

SILJESTRÖM, P. 1985. Geomorfologia y edafopénesis de las arenas del Parque Nacional de Doñana. Tesis Doctoral. Univ. Sevilla. $515 \mathrm{pp}$.

SUSO, J. y R. LLAMAS 1990. El impacto de la extracción de aguas subterráneas en el Parque Nacional de Doñana. Estudios geológicos 46: 317-345.

SUSO, J. and R. LLAMAS 1993. Influence of groundwater development on the Donana National Park ecosystems (Spain). J.Hydrology 141: 239-269.

ter BRAAK, C.J.F. 1987. The analysis of vegetation environment relationships by canonical correspondence analysis. Vegetatio 69: 69-77.

ter BRAAK, C.J.F. 1991. CANOCO v. 3.12. Agricultural Mathematics Group, Wageningen.

TOJA, J.; T. LÓPEZ \& N. GABALLONE 1991. Successional changes in two dune ponds (Doñana National Park). Verh. Internat. Verein. Limnol 24: 1556-1559.

VELA, A. 1984. Estudio preliminar de la hidrogeología e hidrogeoquímica del sistema de dunas móviles y fleclza litoral del Parque Nacional de Doñana. Tesis de Licenciatura. Univ. Complutense. Madrid. 256 pp.

VELA, A., I. HERRÁEZ y J.L. TENAJAS 1987. Relaciones isotópicas entre las aguas superficiales y subterráneas en el área peridunar del Parque Nacional de Doñana. II Congreso de Geoquímica de España. 